\title{
Research on Social Commerce basing on Social Network Analysis
}

\author{
Yingl-iang WU ${ }^{1, a}$, Xin $\mathrm{HU}^{2, \mathrm{~b}^{*}}$, and Jiong-En XIAO ${ }^{3, \mathrm{c}}$ \\ 1,2,3Department of E-Business, School of Economics and Commerce, South China University of \\ Technology, Guangzhou, Guangdong, China \\ aadylwu@scut.edu.cn, bhello.huxin@163.com, cxiaojiongen@qq.com
}

\begin{abstract}
Keywords : social commerce, s-commerce, social network analysis ,e-Business, information management
\end{abstract}

\begin{abstract}
Social commerce(s-commerce), a new and hot area of E-Business which emerged and envolved rapidly in recent years, also the important stage characteristics of the current development and evolution of E-Business. Firstly, We combed the social commerce researches and development systematically. We analyzed the background and motivation, development status and trends of social commerce. Then, we investigated the research and application status of social network analysis. Finally, we discussed the research status of social network analysis in social commerce. We indicated the main problems existed and explained several important development trends and research topics.
\end{abstract}

\author{
基于社会网络分析的社会化商务研究述评 \\ 吴应良 ${ }^{1, a}$, 胡欣 ${ }^{2, b^{*}}$, 肖昫恩 ${ }^{3, c}$ \\ $1,2,3$ 华南理工大学经济与贸易学院电子商务系, 广州, 广东, 中国 \\ aadylwu@scut.edu.cn, bhello.huxin@163.com, cxiaojiongen@qq.com
}

关键词：社会化商务; 社交商务; 社会网络分析; 电子商务;信息管理

中文摘要. 社会化商务（social commerce, s-commerce），又称 “社会化电子商务” ，是 近年来兴起与快速发展的电子商务前沿与热点领域之一, 也是当前电子商务发展与演化的重 要阶段性特征。首先, 本文系统梳理了国内外社会化商务研究与发展脉络, 对社会化商务产 生的背景与动因、发展现状与趋势进行了总结分析; 其次, 考察了社会网络分析 (Social Network Analysis, SNA) 的研究与应用现状。最后对社会化商务研究中的社会网络分析研 究与应用现状做出评述, 指出了存在的主要问题, 并阐述了需要重点关注的几个重要发展动 向与研究课题。

\section{1 引言}

近年来，基于社交网络平台等社会化媒体的电子商务应用势如破竹，催生了 “社会化商 务” (即 “社会化电子商务”)（ social commerce， s-commerce/sCom）这一新兴业态的产生 与快速发展, 并正在衍生出许多新型的商业模式 (Business Model, BM), 引起了学术界的 关注和研究。对企业来说, 消费者的社会化行为与商务活动在各种网络社交平台（社会化媒 体平台）上形成了一类十分丰富的数据信息，在业界被认为是 “价值连城的金矿” ，如何对 这一大量的社会化数据或信息进行必要、有效的分析和管理, 已成为当今企业进行战略决策 需要面对的重大挑战与课题。本文将系统分析社会化商务和社会网络分析（Social Network Analysis, SNA) 两个研究领域的研究进展, 基于 “社会化行为” 这一共同本质特征寻找两者 的结合点, 考察分析 “社会化商务中的社会网络分析” 这一重要研究领域的研究进展、存在 
问题和发展趋势，阐述一些重要的研究议题和思路，包括数据采集、关系识别、商务信息传 播等, 以及如何合理运用SNA方法与工具进行社会化商务中的数据挖掘、知识发现和价值创 造，以发掘社会化商务中潜在的价值，促进社会化商务商业模式的创新。

\section{2 社会化商务研究现状}

\section{1 源起背景及概念界定}

2005年, 雅虎首次引入了 “社会化商务” 标签, 而在学术文献中出现的时间最早是2007 年。而实际上早在1999年，亚马逊Amazon的网站就出现了 “选购圈(purchase circles)” 功能, 和现有的推荐系统和消费者社区功能相同 ${ }^{[6]}$ 。到2006年，国外就已经诞生了如Epinions 、 ThisNext等不错的社会化电子商务公司。2011年, Pinterest以 “瀑布流” 为特征崛起, 国内是 美丽说、蘑菇街、老牌电商和社交平台也相继推出推出微淘、人人迬街、凡客达人等社会化 商务功能。Curty 和 Zhang (2011) 提出了一种定性的纵向研究,通过对社会商务网站中的技 术特性和工具的演化的系统衡量, 来说明它们对社交商务实践的形成和未来发展的影响 ${ }^{6]}$ 。

Wang 和 Zhang (2012) 通过研究社会化商务的演化历程, 从人、管理、技术和信息四个维 度来全面分析社会化电子商务的发展。Turban（2015）认为，社交商务指在Web2.0工具/社交 媒体上开展的电子商务活动, 是电子商务、社媒支持技术和社交媒体内容(群体互动)的结合 ${ }^{[3]}$ 。 Manjit S.（2013）在对已有的概念进行梳理后认为, 在市场上与消费者和公司有关的活动有 着内在的联系，因此社会化商务的领域应该是指同时与消费者和企业相关联的活动 ${ }^{[5]}$ 。虽然 社会化商务被定义的不同，但可以看出，这些定义都显示出了几个共同特征：社媒技术、用 户互动和商务活动。这里, 我们将社会化商务定义为有消费者或企业参与的通过社会化网络 /Web 2.0进行互动和联系的电子商务活动, 是广义的社会化商务。

\section{2 社会化商务的理论研究与应用}

在Web of Science数据库中, 我们以"social commerce"为主题词组来检索所有年份所有数 据库的文献, 发现2007年开始出现相关文献, 2013年出版的文献数量最多, 近两年有所下 降。研究领域分布在社会科学、科学技术和艺术人文三个方面。主要研究国家为美国、中国、 韩国、马来西亚。主要来源期刊排名为Electronic Commerce Research and Applications、 International Journal Of Electronic Commerc和Decision Support Systems, 主要研究方向为计算 机科学和商业经济。若在所有数据库中以四个最常用主题词组: "social commerce" OR "social shopping" OR "collaborative commerce" OR "collaborative shopping"检索, 研究从1999年就开始 有了萌芽(一篇相关文章发表在第4届欧洲多媒体应用会议上)。在2013年达到研究数量最高 峰。在WOS核心合集中, 主要来源期刊排名为: Decision Support Systems、Electronic Commerce Research and Applications、International Journal Of Electronic Commerc, 研究方向依然没变化。

Liang和Turban（2011）认为社交媒体上任何可以导致商业利润的活动都属于社会化商务 活动, 并提出了社会化商务的活动框架, 包括四个方面。分别是：社会化媒体营销、企业社 交商务、技术支持/社交软件整合、管理与组织和其他应用（电子政务、移动商务、社交视频 游戏等）。笔者有观察到很多学者只是简单地将已有的研究进行陈述分类, 并没有相应的逻 辑支持，而Turban的社会化商务活动框架可以有效地指导我们对现有的研究进行梳理。

在技术支持/软件整合方面, 研究主要集中在对社会化商务网站的功能、整体架构的设计 优化、系统集成。例如, Huang Z, 和Benyoucef M (2013) 提出了社会设计的四个核心要素身份、会话、社区和商务 ${ }^{[8]}$ 。Olbrich和Holsing（2011-2012）实验证明了社交购物的功能(如 评级)和标签比直接购物的功能(如笁选和搜索)更能促进交易, 最典型的例子有Polyvore和 ThisNext ${ }^{[9]}$ 。Yung-Ming Li(2014)从用户偏好、地理便捷和朋友的影响这几个方面来考虑设计 了一个团购推荐系统来推广位置感知产品。而在商务应用中，企业将各种Web 2.0特性集成到 他们当前的系统, 极大地提高了商业、社会和协作能力。 
社会化媒体营销层面包含了对商业模式、用户行为、营销策略、品牌建设等发面的研究。 (1)在商业模式方面, Leitner\&Grechenig (2007) 提出四种典型的社会化商务的商业模式: 协作购物网络、生活商务、社区驱动型商务和大规模定制。王宇(2013) 从理论层面探讨了基 于碎片模型的社会化商务价值创造模式, 认为社会网络服务的典型价值网通常形成多层级结 构。一些学者如(MacMillan2011, Lee 2011)指出了一些问题, 现有的商业模式很容易被复制, 行业壁垒不高, 很难避免潜在竞争者的进入。从事社会化商务活动的企业也因为激烈的竞争 被迫花费更多的成本来获取客户和保留客户。而且提供的服务种类过多, 运营成本太高, 导 致收益很低。根据此类问题，Kim（2013）运用NASH均衡博弯模型分析了市场开拓、利润创 造等社会化商务的核心决策问题之间的关系, 并提出了解决当前商业模式缺陷的战略对策和 方向。(2)在用户行为方面, Wigand等(2008)发现了社会化商务中消费者三个基本需求: 成为 独立的个体、有成功和满足感、有归属感。Liang等(2011)发现社会性因素如社会支持、网站 质量和关系质量都对用户持续使用该社交媒体和参与社会化商务活动有积极的影响 ${ }^{[4]}$ 。 Zhang(2014)指出社会化商务中技术特征决定了虚拟用户体验, 而虚拟用户体验又决定了非直 接交易的用户行为。另外, Celeste(2013)探讨了社会网络社区中文化调节作用和信任对社会互 动和购买意愿之间关系的中介作用。(3)在营销策略方面, Wang \& Zhang (2012) 指出可以通 过将现有的媒体渠道进行整合和拆分来获得启发, 例如可能需要简化购物渠道, 进行媒体、 内容的整合，或者开发利基市场。Toubia和Stephen (2013) 在对Twitter用户的调查中发现，社 交媒体如Twitter可能作为社会化商务的广播渠道，比消费者追踪渠道更加有用 ${ }^{[8]}$ 。相对于企 业提供的信息，消费者更信赖同伴们的使用信息反馈。因此社会化商务网站可以通过动态影 响企业运作效率、效果、客户关系、产品服务供给和利润增长来帮助企业增强竞争优势，而 不仅仅是简单的电商和社交的技术结合。Laroche et al.指出了建立品牌社区对消费者价值创 造、品牌信任和品牌忠诚的积极作用，有利于广告和其他营销推广。

此外，关于企业社交商务、管理与组织和其他应用这三个方面，研究文献则相对较少。 相关概念尚未确定, 目前对企业社会化商务的研究停留在协作系统设计等技术层面, 这在前 面的技术层面已有阐述。除了Turban ${ }^{[3]}$ 的介绍, 国内外几乎没有较好的研究成果。

\section{3 社会网络分析研究现状}

\section{1 社会网络分析的理论研究}

当代社会网络分析可追溯到20世纪30年代, 由不同学派学者的研究相互影响而发展起来, 并在1960年代和1970年代出炉于哈佛。1922年，人类学家布朗Radcliffe Brown以 “相对非技 术化的形式” 首次提出 “结构功能论” 概念,经历了 “隐喻”、 “形式化” 和 “概念化 和技术化”各个阶段。社会网络分析的发展主要有三条发展主线，如图3所示。

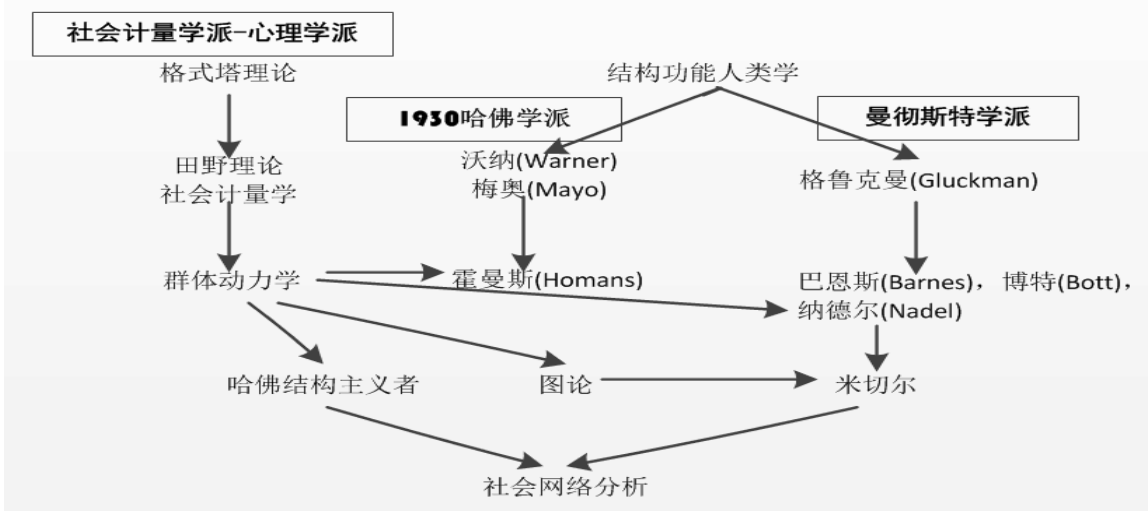

图 1 社会网络分析发展谱系图

在这三个群体的研究基础上, 怀特( Harrison White)领导的哈佛学派扩展了对社会结构的 数学基础的研究，提出了 “块模型” ，使得社会网络分析真正成为一种结构分析的方法。关 键在于群体代数模型的发展和多维量表的发展。斯科特认为, 社会网络分析是一种面向自身 
且有着特定方法的社会世界理论取向。如今，Burt、Granovetter、Freeman、HarrisonWhite等 是这一领域的重要贡献者。其中, 社会网络分析的著名理论有: 小世界网络、尺度无关网络, “机会链” 理论、网络结构理论、弱关系力量假设与强关系理论、“嵌入性”、“社会资本”、 “结构洞” 等。社会网络是人类社会关系特征的突出表现形式, 而社会网络分析不仅是一种 研究方法, 还是一种研究社会结构关系的新观点。它关注的主要问题是社会互动的形式化特 征, 研究对象就是社会结构, 表现形式为行动者之间的关系模式。但也有学者指出, 这一定 程度上忽略了节点的能动性, 并且, 在界定关系时也未能挖掘出能动者在相互 “社会支持” 背后蕴含的丰富的 “文化” 要素。

刘军指出，当代社会化网络领域中取得重大突破性进展的大多出现在整体网中。社会网 络分析常用的分析方法有: 图论(graph theory)、社会计量学和代数方法、统计模型。其中, 图论以点和线的形式表示行动者及其关系，适用于描述小群体的核心关系和团聚力等特征; 社会计量学适用于研究结构等价性和 “块模型” ; 代数法适用于对群体关系、角色和位置关 系进行分析。常用的软件和处理工具有: UCINET、Pajek、NetMiner、NetDraw、STRUCTURE、 GRADAP等, 其中综合型分析软件UCINET的使用最为普遍, 以简单的矩阵形式存储数据, 可以与多种软件进行数据交换。功能最强大的是GRADAP, 可以直接与SPSS交换数据, 要求 把领结矩阵分为独立的 “点文件” 和 “线文件” , 目的是将关系数据彻底转换成图论术语, 这时需要使用者完全掌握图论知识。

\section{2 社会网络分析的应用研究}

计算机技术极大地促进了社会网络分析的发展，使得社会网络分析(SNA)从社会学迅速 扩展到其他领域, 如政治学、管理学、人类学、心理学等。主要集中在信息传播、组织管理、 知识管理等领域。国外学者更多的是将社会网络分析应用于社会中群体的年龄结构、行为习 惯特征、宗教信仰、劳动力市场流动、社会关系、疾病的产生蔓延以及医疗对策等社会现象, 而国内侧重对不同社会群体中关系网络的构建与可视化等方面的研究，如组织行为与管理、 人际关系与网络社区、文献计量等。

中国作为一个关系社会, 研究社会关系的属性、结构和演变对于更好地认识中国社会的 发展、变迁和创新社会治理模式具有重要意义。而社会网络分析方法在揭示社会机制作用过 程上有显著优势, 因此很多学者对各种社会问题进行了质性研究和量化探讨, 如Ruobing(2015) 用SNA评估了跨文化交流能力(ICC)。任义科等（2011）分析了深圳市农民工社会支持和讨论 网络的宏微观结构, 探讨了农民工社会关系的深层次结构特点和社会融合等问题。而大多数 学者聚焦于对信息传播、與情研究、意见领袖、行为特征等方面各种视角的研究, 比如在突 发事件上的应用。赵金楼和成俊会(2015) 以2013年 “4. 20 四川雅安地震” 为例, 运用 SNA 对突发事件微博舆情传播网络结构特征进行了分析与测量。此外, 国内外学者还将SNA应用 到人口迁徙（王珏2014）、公众健康(Martin2014)、自然资源管理(Riikka2015、骆耀峰2015)、 反恐(李本先2012)、国际战争冲突(Olga\&Robert2013)、城市网络信息结构(陈映雪2013)、地缘 政治(潘峰华2013)、航空探测沟通(Harry2013)等。

组织管理方面，研究一般集中在知识管理、创新管理、供应链管理、产业集群、企业集 群、连锁董事、合作网络等方面。(1)应用在组织内部可以进行知识共享, 帮助发现问题, 提 高企业竞争能力。如赵英(2014)运用SNA对部门间、部门内使用社交媒体所形成的各种非正 式网络进行对比分析, 发现对社交媒体的合理使用可以提高企业知识创新能力。李平(2008) 采用矩阵法收集数据并利用Pajek软件计算矩阵的接近中心度来识别企业中关键智力资本。曾 勇(2012)用SNA 探测企业内部隐藏的犯罪活动, 运用模糊评价模型对所有员工进行嫌疑程度 的综合评价。另外, 还能研究员工工作满意度、离职倾向等群体心理行为。通过建立网络动 力学模型还可以预测组织演化的趋势。(2)而将SNA应用在组织间网络, 可以帮助组织进行自 我定位和竞争、合作战略管理。如Sankar等(2015)分析了印度国家政权交易所里的企业中连锁 董事网络结构特征, 证实了企业间小世界网络的存在。Tiffany等(2014)对纽约的海洋生态系 统管理的协作网络进行案例研究, 探讨其协作机制的运转过程。邵云飞(2009)归纳了国内关 
于企业集群创新网络的一些实证研究, 并建议未来研究能够将SNA与案例研究、传统计量经 济学结合在一起。另外, 张树人还指出通过具体案例分析可以帮助发现某个特定的企业集群 的形成机制(如小世界理论与知识扩散的结合)，继而自觉培育特色区域经济。

此外, 社会化网络分析还经常被用于对某一研究领域文献计量、合著网络的网络结构探 讨。如Choi(2014)将UCINET和NetDraw分别用来对韩国教育技术研究领域的关键字提炼和可 视化, 从而挖掘发展主题和趋势。李亮(2008)对国内情报学领域的合著网络进行定量研究, 揭示现状和问题。

\section{4 社会化商务中的社会网络分析：研究述评}

社会化商务研究还处于较为初级的阶段, 对主要问题的解释也仅仅停留在理论层面, 缺 乏社交网络和社交媒体基础上的商业价值的实证研究。目前国内外关于社会化商务中社会网 络的分析主要分布在对社交网络中用户行为、信息传播、功能设计、个性化推荐等方面在电 商上的延伸, 细化了调查对象。田雨晴 (2013) 指出, 大多数研究都只是单纯对社会网络进 行分析, 而没有从同电子商务的角度结合起来, 缺乏对社会化电子商务效果的研究。可以考 虑从信息经验分享和网络关系角度来探讨。如王佳秋（2013）采用网络爬虫和新浪应用程序 接口相结合的方式, 收集新浪微博品牌馆中微博电商部分用户作为研究对象, 对电商用户社 交关系进行聚类从而提出新的检测用户节点影响力的计算方法, 并用Gephi加以可视化。丁绪 武等(2015)通过改进的用户兴趣建模方法和可视化复杂网络分析工具 Gephi 构建美丽说上用 户的兴趣图谱。ai-Yu(2013)利用UCINET和Web挖掘技术分析和发现虚拟社区中的利益群体, 构建推荐系统。

杨玉洁（2014）采用结构方程模型，根据调查数据检验了社交网络属性对电商消费者信 任的影响。武鹏飞（2015）构建了在线评论相关因素与消费者对社交网络中电子口碑采纳行 为的影响关系。易明等(2010)利用SNA构建挖掘社会化标签的内在逻辑和构建web用户的局部 兴趣视图, 而后(2014)又研究了社会化标签系统中的知识传播网络演化过程, 利用UCINET构 造拓扑图, 并用NetDraw加以可视化, 从而进行实证研究。可以看到, 这部分的研究处于起 步的状态, 很多方面比如社交电商内容、电商企业营销效果、品牌影响、公关等方面都未做 出相应研究, 值得进一步探讨和研究。

综合以上分析, 我们认为, 值得重点关注的发展方向和课题可以有: (1)考虑采用实证(如 实验)、仿真等) 方法对社会化电子商务的商业模式和平台设计进行深入的研究, 考虑如何让 商家和消费者在网络社交关系维系中, 自然地进行交易活动。考察在社会化情境下商业模式 要素的演变、重构价值链, 发展可持续的商业模式, 进一步为实践者提供理论指导。(2)关注 和结合实践者的需求, 构建具有普适性的、适合社会化电子商务的理论或模型, 拓展研究的 深度。如社会化电商平台的营销评价体系的建立、数据的利用等, 对企业运营有现实指导意 义。(3)开发社会化网络的更多功能使得电子商务能更好地利用现有的资源, 利用独特的组织 能力, 和支持有价值的客户关系。(4)关注社会化电商平台不同角色的社会化特征, 如企业组织 管理、竞争分析。(5)尝试与其他研究领域相结合, 扩展研究范围, 从不同角度分析社会化电 子商务的商业价值及社会价值, 比如社会化电子商务对旅游业、电影业、对政府服务的影响 等。

本文对国内外社会化电子商务最新的研究成果进行了系统地梳理, 对存在的主要问题给 出了相应的建议, 为后续研究该领域的学者奠定了基础, 但仍有很多不足。社会化电子商务 是个实践性非常强的领域, 也能创造比传统营销模式更丰厚的利润、更多样化的营销方法, 提高用户体验。因此值得更多的学者在社会化电子商务领域深耕细作, 为社会创造更多的价 值。 


\section{致谢}

本文为国家自然科学基金重大资助项目《网络环境下的服务创新与服务设计研究》(71090403/71090400);

国家社会科学基金项目 “分享经济下基于 TRIZ 理论的网络约租车服务创新研究” (16BGL190); 国家社会 科学基金项目 “大数据背景下金融市场信用违约预测和违约风险补偿研究”（15BJY149）的阶段性成果之

\section{References}

[1] Stephen A T, Toubia O. Deriving value from social commerce networks[J]. Journal of marketing research, 2010, 47(2): 215-228

[2] Marsden P. Commerce gets social: How your networks are driving what you buy[J]. Social Commerce Today, 2011

[3] Turban et al. Electronic Commerce: A Managerial and Social Networks Perspective(Eighth Edition)[M]. USA: Springer International Publishing Switzerland, 2015

[4] Liang, Ting-Peng, Yi-Ting Ho, Yu-Wen Li, and Efraim Turban, What Drives Social Commerce: The Role of Social Support and Relationship Quality[J], International Journal of Electronic Commerce, 2011,16(2):69-90

[5] Manjit S. Yadava. Social Commerce: A Contingency Framework for Assessing Marketing Potential[J]. Journal of Interactive Marketing, 2013, (27): 311 314

[6] Curty R G, Zhang P. Social Commerce: Looking Back and Forward [C]. Proceedings of the American Society for Information Science and Technology ( ASIST ) Annual Conference. USA:New Orleans, 2011,48(1):1-10

[7] Huang Z, Benyoucef M. User preferences of social features on social commercewebsites: An empirical study[J]. Technological Forecasting \& Social Change, 2015,(95):57-72

[8] Toubia, Olivier and Andrew T. Stephen. Intrinsic Versus Image-related Utility in Social Media: Why Do People Contribute Content to Twitter? [J]. Marketing Science, 2013, 32(3):368-92

[9] Olbrich, Rainer and Christian Holsing. Modeling Consumer Purchasing Behavior in Social Shopping Communities with ClickstreamData[J]. International Journal of Electronic Commerce, 2011-12, 16(2): 15-40 